\title{
Sleep hypoxia in myotonic dystrophy and its correlation with awake respiratory function
}

\author{
A J Finnimore, R V Jackson, A Morton, E Lynch
}

\begin{abstract}
Background - Tiredness and daytime respiratory failure occur frequently in myotonic dystrophy. Sleep hypoxaemia was studied in 12 patients with myotonic dystrophy and correlations were sought with their daytime lung and respiratory muscle function.
\end{abstract}

Methods - All patients underwent overnight sleep studies, clinical assessment, measurement of flow-volume loops and carbon monoxide transfer factor, arterial blood gas analysis, and physiological assessment of both thoracic muscle function and upper airways obstruction.

Results - The mean nadir of oxygen saturation during sleep was $75 \%$ (95\% confidence interval $69 \%$ to $81 \%$ ). A mean of $3.4 \%$ of total sleep duration was spent at an oxygen saturation level below $85 \%$. Five of the 12 patients had an apnoea index of $>5$, the group mean apnoea/ hypopnoea index being 15.8 events/sleep hour. The mean awake arterial oxygen tension $\left(\mathrm{PaO}_{2}\right)$ was $10.7 \mathrm{kPa}$. There was a trend to hypercapnoea with a mean awake arterial carbon dioxide tension of $6.1 \mathrm{kPa}$; carbon dioxide retention worsened during sleep. Respiratory muscle dysfunction was mainly evident as a low maximum expiratory mouth pressure. Upper airway obstruction assessed by physiological criteria was found in four of the 12 patients. The proportion of total sleep duration with oxygen saturation levels below $85 \%$ was directly related to body mass index (weight/height ${ }^{2}$ ) and inversely related to the awake $\mathrm{PaO}_{2}$. Body mass index was inversely related to the overnight nadir of oxygen saturation. Conclusions - Patients with myotonic dystrophy are often hypoxic during sleep and the subgroup that are obese, or have symptoms of sleep apnoea, or both, are particularly at risk. Sleep studies should be considered in this subgroup of patients with myotonic dystrophy.

(Thorax 1994;49:66-70)

Myotonic dystrophy is an autosomal dominant, multisystem disease affecting skeletal, smooth, and cardiac muscle as well as causing nervous system abnormalities, cataracts, and endocrine deficiencies. It is the commonest muscular dystrophy affecting both children and adults, and has a prevalence of about one in 8000-10 000 in the population. ${ }^{1}$ Three studies have examined the prevalence and effect of sleep hypoxia in 19 randomly selected patients with myotonic dystrophy. ${ }^{2-4}$ It is not standard practice to test all patients with neuromuscular disease for sleep hypoxia, and the incidence of sleep hypoxia and its long term significance in this group are not known. A recent paper studied sleep in various neuromuscular disorders and recommended screening for sleep disordered breathing. ${ }^{3}$

Our study set out to examine sleep hypoxia and awake respiratory function in a group of patients with myotonic dystrophy who had not presented with a sleep disorder. Daytime arterial blood gas tensions, respiratory muscle strength, upper airway physiology, and obesity were all studied to investigate possible correlations with a sleep disorder.

\section{Methods}

Twelve consecutive patients presenting to the Neuroendocrine Research Unit with clinically and electrophysiologically confirmed myotonic dystrophy were studied. Subject selection was independent of sleep disorder symptoms. There were eight men and four women of mean age 41 (range 25-70) years (table). Four
Chest Clinic A J Finnimore E Lynch

Neuroendocrine Research Unit R V Jackson A Morton

The University of Oueensland Department of Medicine, Greenslopes Hospital, Brisbane, Queensland 4120, Australia

Reprint requests to: Dr R V Jackson.

Received 8 February 1991 Accepted for publication 22 September 1993
Clinical characteristics of 12 patients with myotonic dystrophy

\begin{tabular}{|c|c|c|c|c|c|c|}
\hline Age (years) & Snorer & Sleep apnoea sequelae & Alcohol/sedatives & Disability & Lung disease & Cigarettes (packs/year) \\
\hline $\begin{array}{l}32 \\
62^{*}\end{array}$ & Mild & $\begin{array}{l}\text { Hypersomnolent } \\
\text { witnessed apnoeas }\end{array}$ & & Limp & Lung abscess & 1 \\
\hline $\begin{array}{l}54 \\
37 \\
36\end{array}$ & Mild & & $\begin{array}{l}20 \mathrm{~g} / \text { week } \\
70 \mathrm{~g} / \text { week } \\
20 \mathrm{~g} / \text { week }\end{array}$ & Limp & Asthma & \\
\hline $\begin{array}{l}25 \\
33\end{array}$ & & & $20 \mathrm{~g} /$ week & & & $\begin{array}{l}2 \\
5\end{array}$ \\
\hline 70 & & Mane headache & $\begin{array}{l}\text { Temazepam } \\
20 \mathrm{mg} / \text { day }\end{array}$ & $\begin{array}{l}30 \mathrm{~m} \\
\text { walking }\end{array}$ & & 70 \\
\hline $\begin{array}{l}31 \\
51\end{array}$ & Yes & & $50 \mathrm{~g} /$ week & & Pneumonia & 17 \\
\hline $\begin{array}{l}37 \\
27\end{array}$ & Mild & & $60 \mathrm{~g} /$ week & $\begin{array}{l}\text { Invalid } \\
\text { pension }\end{array}$ & Pneumonia & 25 \\
\hline
\end{tabular}

\footnotetext{
* An index case of myotonic dystrophy.
} 
were current smokers and two were exsmokers. Body mass index (BMI = weight/ height ${ }^{2}$ ) ranged from 17 to 31 (group mean 23) with the ideal index being 20-25. All patients were clinically euthyroid, and all 10 patients tested were biochemically euthyroid.

\section{STUDY DESIGN \\ Awake tests}

Clinical assessment included inspection of the pharynx for narrowness, voice quality, and historical features of sleep apnoea. These were scored before any physiological results were known. The symptoms enquired about were: snoring, hypersomnolence, witnessed apnoeas, morning headache, and daytime nap tendency.

Respiratory function tests (spirometric measurements, flow-volume loops, single breath carbon monoxide transfer factor (TLCO), and maximal voluntary ventilation for 12 seconds $\left(\mathrm{MVV}_{12}\right)$ ) were performed to American Thoracic Society criteria. The spirometer used to record flow-volume loops and MVV was a Vitalograph Compact recalibrated before each testing session. Reference ranges for flow-volume data were those of the European Coal and Steel Community. ${ }^{5}$ TLCo was measured on a Jaeger Masterlab using reference values from Viljanen et al. ${ }^{6}$

Arterial blood gas analyses were performed on the average of two room air samples taken whilst awake and seated, five minutes after local skin infiltration with $1 \%$ lignocaine. One sample was collected the night before and the other on the morning after the sleep study. A Radiometer (Copenhagen) ABL300 blood gas analyser was used. One patient refused blood gas sampling.

Respiratory muscle weakness was assessed by three parameters:

(1) Maximum inspiratory pressure at residual volume (MIP) and maximal expiratory pressure at total lung capacity (MEP) were tested on Magnehelix instruments using the reference values of Wilson et al. ${ }^{7}$

(2) Percentage fall in forced vital capacity supine $v$ standing $(\triangle F V C)$ is an index of diaphragm dysfunction. ${ }^{8} \Delta \mathrm{FVC}$ of $>25 \%$ was considered abnormal in the absence of obstructive airways disease.

(3) Maximum voluntary ventilation over 12 seconds $\left(\mathrm{MVV}_{12}\right)$ reflecting net respiratory muscle strength and endurance ${ }^{9}$ was determined from the best of two trials to minimise patient distress. Predicted values were those of the European Coal and Steel Community. ${ }^{5}$

Upper airway obstruction was assessed using previously validated techniques. ${ }^{10-12}$ Obstruction was considered to be present if at least two of the following four criteria were met:

(1) Ratio of forced expiratory flow at 50\% vital capacity to forced inspiratory flow at $50 \%$ of vital capacity $\left(\mathrm{FEF}_{50} / \mathrm{FIF}_{50}\right) \geqslant 1$.

(2) Ratio of forced expiratory volume in one second $\left(\mathrm{FEV}_{1}, \mathrm{ml}\right)$ to peak flow (PEF, 1/ $\min ) \geqslant 8.5$.
(3) Peak inspiratory flow (PIF) $<31 / \mathrm{min}$.

(4) Helium response: ratio of PEF breathing helium to PEF on air $>1.5$ measured after three vital capacity breaths of a mixture of $80 \%$ helium and $20 \%$ oxygen. ${ }^{13}$

The flow-volume curves were also inspected for abnormal contours - for example, plateaux or irregular abrupt changes in flow suggesting intermittent airway obstruction. ${ }^{10}$ These physiological tests were performed within four weeks of the relevant sleep studies.

\section{Sleep data}

Sleep data were collected during an overnight inpatient stay in a single room.

Vitalog computerised equipment Version 4.2 (Vitalog Corporation, California, USA) allowed recording of (1) oxygen saturation using a Biox Ohmeda 3700 oximeter; (2) pulse rate from a three lead ECG; (3) thoracic and abdominal excursion via impedance plethysmography; and (4) arm movements. Apnoeas were scored by computer when absent inspiratory movements were present for $\geqslant 15$ seconds. Hypnoeas were scored when inspiratory excursions were less than two thirds of the concurrent baseline value for at least 15 seconds. There is a strong correlation between this method of identifying hypopnoeas and the 10 second event as scored during polysomnography (Vitalog). Obstructive events were scored when thoracic and abdominal respiratory traces detected paradoxical movements. A validation study of 12 patients by Gyulay et al showed a good correlation of apnoea/hypopnoea index values between a polygraph and a Vitalog system $(r=0.70, \mathrm{p}<0.01)$ and all patients with sleep apnoea were correctly diagnosed by the Vitalog system. ${ }^{14}$

Transcutaneous carbon dioxide pressures were measured throughout the study night (TCC3 apparatus; Radiometer, Copenhagen, Denmark) and baseline and peak nocturnal levels were read off the resultant printout.

Patient diaries were kept and at least four hours of sleep were recorded in all patients.

\section{STATISTICAL ANALYSIS}

Statistical analysis was by unweighted least squares linear regression or by rank sum (two sample) Mann-Whitney tests. The significant associations were also reanalysed by Spearman rank correlations as this presenting sample may not be normally distributed. All data are expressed as mean values with $95 \%$ confidence intervals.

\section{Results}

SLEEP DATA

Nocturnal hypoxia was noted. The mean nadir of oxygen saturation reached during sleep was $75 \%$ ( $95 \%$ CI $69 \%$ to $81 \%$ ). Oxygen saturation was below $85 \%$ for a mean of $3.4 \%$ $(0.4 \%$ to $6.4 \%)$ of total sleep duration. The mean number of episodes with a $4 \%$ fall in oxygen saturation was $18(95 \%$ CI 10.5 to 

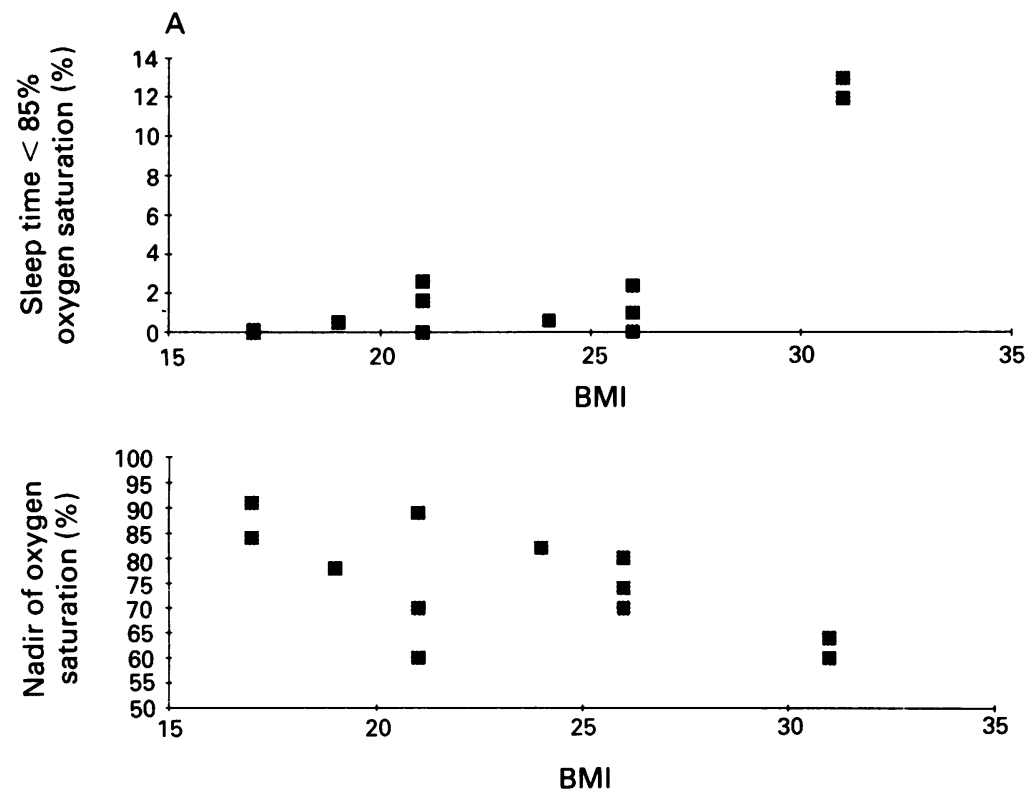

Figure 1 Body mass index (BMI) plotted against $(A)$ percentage of sleep time spent below $85 \%$ oxygen saturation and $(B)$ nadir of overnight percentage oxygen saturation. BMI correlated significantly with sleep time below $85 \%$ oxygen saturation and with the nadir of oxygen saturation $(p<0.005$ and $p<0.05$, respectively).

25.4) per sleep hour. Two patients had relatively prolonged periods of hypoxia with saturations below $85 \%$ for $12.9 \%$ and $11.9 \%$ of the sleep time; another two patients did not desaturate.

The mean apnoea index was 6.4 per hour, and the mean apnoea/hypopnoea index was $15 \cdot 8(10-21 \cdot 6)$ events/sleep hour. Five of the 12 patients had more than five apnoeas/sleep hour. Obstructive apnoeas were suggested by paradoxical respiratory efforts in $72 \%$ of all events and in $61 \%$ of events in those with $>5$ apnoeas/hour (range $39 \%-73 \%$ of events).

BMI correlated significantly (least squares regression analysis) with the nadir of oxygen saturation $(p<0.005)$ and the Spearman rank correlation for BMI and nadir of oxygen saturation was $0.62(95 \% \mathrm{CI} 0.07$ to 0.88

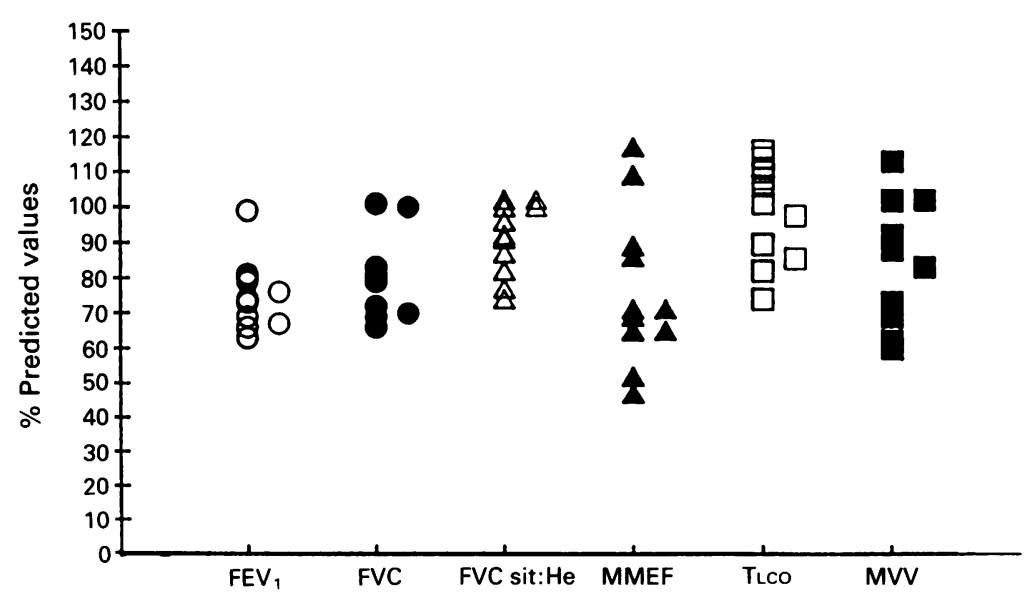

Figure 2 Awake lung function tests in 12 patients with myotonic dystrophy plotted against the percentage predicted value of these variables. The symbols offset to the right in each category represent the two patients who did not desaturate below $90 \%$ overnight. $F E V_{1}=$ forced expiratory volume in one second; $F V C=$ forced vital capacity; FVCsit:He = ratio of FVC on air to FVC on $80 \%$ helium when seated; $M M E F=$ maximum mid expiratory flow rate from $25 \%$ to $75 \%$ of vital capacity; TLCO = transfer factor for carbon monoxide; $M V V=$ maximum voluntary ventilation over 12 seconds. Group mean values are: FEV, 74\% predicted, FVC $80 \%$ predicted, FVCsit:He ratio 0.91 , MMEF $76 \%$ predicted, TLCO $100 \%$ predicted, MVV $82 \%$ predicted. $\mathrm{p}<0 \cdot 05)$. BMI also correlated significantly (least squares regression analysis) with the number of $4 \%$ desaturations recorded $(\mathrm{p}<0.05)$, with a Spearman rank correlation coefficient of 0.64 (95\% CI 0.11 to 0.89 ; $\mathrm{p}<0.05)$. There was a highly significant but non-linear correlation of BMI with sleep time spent below $85 \%$ oxygen saturation $(\mathrm{p}<0.005)$, with a Spearman rank correlation coefficient of 0.65 ( $95 \%$ CI 0.12 to 0.89 ; $\mathrm{p}<0.05$ ) (fig 1). BMI did not predict the apnoea/hypopnoea index.

ARTERIAL BLOOD GAS TENSIONS

All patients had arterial oxygen tensions above $8 \mathrm{kPa}$ while awake with a mean $\mathrm{PaO}_{2}$ of $10.7 \mathrm{kPa}$ (range 9.9-11.6). Awake carbon dioxide retention with $\mathrm{PaCO}_{2}>6 \mathrm{kPa}$ was found in six of the 11 patients tested. The mean $\mathrm{PaCO}_{2}$ while awake was $6 \cdot 1 \mathrm{kPa}(5 \cdot 8-6 \cdot 5)$. Overnight mean transcutaneous carbon dioxide pressure rose to $7 \cdot 7 \mathrm{kPa}$ from the awake value of $6.7 \mathrm{kPa}$. Transcutaneous carbon dioxide readings while awake were higher than concurrent arterial $\mathrm{PaCO}_{2}$ values. Nocturnal carbon dioxide retention is not significantly correlated with awake $\mathrm{PaCO}_{2}$. The awake transcutaneous carbon dioxide value did predict the peak nocturnal carbon dioxide $(\mathrm{p}<0.01)$.

By least squares regression analysis awake $\mathrm{PaO}_{2}$ predicted the sleep duration spent at $<85 \%$ saturation $(\mathrm{p}<0.05)$ and also the number of $4 \%$ drops in oxygen saturation recorded $(p<0.05)$. Spearman rank correlation of awake $\mathrm{PaO}_{2}$ and sleep duration spent at $<85 \%$ oxygen saturation was $0.85(95 \% \mathrm{CI}$ 0.48 to $0.96 ; \mathrm{p}<0.01$ ); for awake $\mathrm{PaO}_{2}$ and the number of $4 \%$ drops in saturation it was 0.78 ( $95 \%$ CI 0.30 to $0.95 ; \mathrm{p}<0.02$ ). No significant correlations were detected between awake $\mathrm{PaO}_{2}$ and apnoea/hypopnoea index and nadir of oxygen saturation, respectively.

Routine respiratory function tests showed mild impairment for all parameters except MEP, which was severely impaired. Results expressed as the mean percentage of the values predicted on the basis of age, sex and height were: $\mathrm{FEV}_{1} \mathbf{7 4 \%}(\mathbf{6 8 \%} \mathrm{-80} \%)$, FVC $\mathbf{8 0} \%$ $(74 \%-86 \%), M_{12} 82 \%(72 \%-92 \%)$, MIP $84 \%(62 \%-106 \%)$, and MEP $32 \%(23 \%-$ $41 \%$ ). Carbon monoxide transfer factor, however, was normal with a mean of $100 \%$ predicted $(90 \%-109 \%$ ) (fig 2$)$. None of the respiratory function tests was predictive of sleep disordered breathing, awake hypercapnoea, nor of a rise in transcutaneous carbon dioxide pressure during sleep.

Only one of the 12 patients had diaphragm weakness suggested by an FVC value of $>25 \%$. This patient was normocapnoeic while awake and had little sleep hypoxia.

\section{UPPER AIRWAY OBSTRUCTION}

The pharynx was narrow on inspection in four of the 12 patients, and a high pitched, nasal voice was evident in five. Five reported dysphagia. Physiological findings suggestive of upper airway obstruction was found in four of 
the 12 who met at least two of the four criteria outlined in the methods section; this subgroup all had sleep hypoxia but the correlation with these physiological variables was not significant. The shape of the flow-volume curve was abnormal with plateaux in four of the patients; this did not correlate with any measure of sleep disordered breathing. (Different individuals were in each of the subgroups listed.)

\section{Discussion}

The major findings in this study are: (1) that sleep disordered breathing with hypoxia is common in patients with myotonic dystrophy, (2) few patients have symptoms of this, and (3) hypercapnoea is observed while awake and worsens overnight. The mean nadir of oxygen saturation of the group was $75 \%$ with 10 of the 12 patients becoming hypoxic overnight. Body mass index and awake $\mathrm{PaO}_{2}$ were predictive of nocturnal hypoxaemia.

A history suggesting sleep apnoea - for example, hypersomnolence or witnessed apnoeas - was given by only one of the group, yet five had an apnoea index of $>5$. Four others were snorers. Lack of acclimatisation and possibly poor quality sleep may have influenced our results.

A recent paper by Bye $e t a l^{\beta}$ reported data on a subgroup of four patients with myotonia who were less well than our group with more hypercapnoea (mean awake $\mathrm{PaCO}_{2} 7 \cdot 0 \mathrm{kPa}$ ) and lower overnight nadir of oxygen saturation (mean 63\%). Our study and that of Bye et al show a significant correlation of the nadir of oxygen saturation with BMI and also with awake $\mathrm{PaO}_{2}$. Analysis of the full group of patients with neuromuscular dysfunction in the study by Bye et al showed additional correlates of nadir of oxygen saturation with vital capacity and also with $\triangle F V C$; neither were demonstrable in our group. Alcohol intake was not likely to contribute to sleep disordered breathing, the mean intake being 20 $\mathrm{g} /$ week with a maximum of $70 \mathrm{~g} /$ week.

Sudden death is a recognised complication of myotonic dystrophy and is not confined to those with advanced muscle disease. These deaths are often linked to ventricular arrhythmias $^{1}$ and nocturnal hypoxia is a risk factor for these arrhythmias. ${ }^{15}$ Intervention with nasal continuous positive airway pressure is warranted in cases of obstructive apnoea showing arterial oxygen desaturation below $75 \% .{ }^{15}$ The mean nadir of oxygen saturation in our group was $75 \%$.

The only clinical feature we found to correlate with sleep hypoxaemia was obesity. Body mass index was significantly associated with the nadir of oxygen saturation, time spent at saturations below $85 \%$, and the number of $4 \%$ drops in oxygen saturation. In contrast, the most sleep disordered cases in the study by Gilmartin et al were not the most obese. ${ }^{4}$ These workers showed that myotonic dystrophy was a major risk factor for sleep disordered breathing. They used non-myotonic controls with neuromuscular disease, but the myotonic cohort was more obese than the controls with a mean BMI of 28 (range 25-32) $v 23$ (range 16-41) respectively. The patients in our study had a mean BMI of 23 (range 1731 ). Both of the patients with nadir oxygen saturation values of $60 \%$ and $70 \%$ respectively had normal BMI values of 21 .

Only one of our 12 patients had hypersomnolence and witnessed apnoeas and his nadir of oxygen saturation was $82 \%$ with an apnoea/ hypopnoea index of 21 events/sleep hour, these being near the means for our group. Ellis et al have noted that patients with neuromuscular disease may function extremely well in the daytime despite major degrees of obstructive apnoea and severe desaturations during sleep. ${ }^{17}$

Respiratory function tests showed a similar pattern to those in previous studies. ${ }^{18}{ }^{19} \Delta \mathrm{FVC}$ suggested diaphragm weakness in one of 12 in our study compared with one of 19 tested by Serisier et al. ${ }^{17}$ Our study found no correlation between indices of respiratory muscle weakness and awake hypercapnoea in agreement with Serisier's earlier conclusions. ${ }^{17}$

This study shows a trend to hypercapnoea but the aetiology is not clear. Bye et al suggested that sleep associated hypoventilation as a result of the neuromuscular disease may be a determinant of awake $\mathrm{PaCO}_{2}$. Our study showed both sleep hypoventilation and nocturnal increases in $\mathrm{PaCO}_{2}$, but did not find any significant relation between awake $\mathrm{PaCO}_{2}$ and sleep disordered breathing.

Paradoxical abdominal movements were detected in $72 \%$ of the apnoeas and hypopnoeas, implying mostly obstructive or mixed sleep apnoeas. Formal polysomnography would distinguish central from obstructive apnoeas more clearly. Upper airway parameters did not correlate with sleep disordered breathing; however, the situation when asleep and supine may differ. Polysomnographic studies have shown a mostly central pattern: six of eight myotonic patients studied by Cirignotta $e t a l^{2}$ had a sleep apnoea syndrome which was central in all cases. Gilmartin $e t a l^{4}$ found a sleep apnoea syndrome in three of seven patients which was central in aetiology in two cases and obstructive in one.

Patients in our group who probably need intervention are those with definite symptoms, prolonged periods spent at low oxygen saturations, and one subject who suffered concurrent moderately severe ischaemic heart disease. Weight control and advice to avoid sedatives seems prudent for all.

Our results suggest that sleep studies may be warranted in patients with myotonic dystrophy, particularly in those who have waking hypoxia or are significantly obese.

The authors thank Drs B Hickey and C Schull for their help in reviewing the manuscript, and Myrna Barnfield for excellen secretarial assistance.

This work was supported by grants from the $\mathrm{CH} \& \mathrm{MRC}$ Australian Department of Veterans' Affairs and the Ramaciotti Foundations.

1 Harper PS. Myotonic dystrophy. 2nd edn. Philadelphia: Saunders, 1989.

2 Cirignotta F, Mondini S, Zucconi M. Sleep related breathing impairment in myotonic dystrophy. $f$ Neurol 1987;235:80-5.

3 Bye PT, Ellis ER, Issa FG. Respiratory failure and sleep in neuromuscular disease. Thorax 1990;45:241-7. 
4 Gilmartin J, Cooper B, Griffiths C. Myotonic dystrophy and non myotonic muscle weakness. $Q \mathcal{F}$ Med 1991;78:2131 .

5 Quanjer PH (ed). Standardised lung function testing. Report of Working Party of the European Community for Coal and Steel. Bull Eur Physiopathol Respir 1983; Supplement.

6 Viljanen AA, Viljanen BC, Halttunen PK, Kreus KE Pulmonary diffusing capacity and volumes in healthy adults measured with the single breath technique. Scand $\mathscr{f}$ Clin Lab Invest 1981;41(Suppl 159):21-34.

7 Wilson SH, Cooke NT, Edwards RH. Predicted normal values for maximal respiratory pressures in Caucasian values for maximal respiratory pressures
adults and children. Thorax 1984;39:535-8.

8 Allen SEM, Hunt B, Green M. Fall in vital capacity with posture. Br $\mathcal{F}$ Dis Chest 1985;79:267-71.

9 Cherniack RM. Pulmonary function testing. 2nd edn. Philadelphia: Saunders, 1992 .

10 Vincken WG, Gauthier S, Dollfuss RE, Hanson RE. Involvement of upper airway muscles in extrapyramidal disorders. N Engl 7 Med 1984;311:438-42.

11 Kryger M, Bode F, Antic R. Diagnosis of obstruction of the upper and central airways. Am ₹ Med 1976;61:85-93.

12 Rotman H, Liss H, Weg J. Diagnosis of upper airway obstruction by pulmonary function testing. Chest 1975;68:796-9.

13 Despas P, Leroury M, Macklem P. Site of airway obstruction in asthma as determined by maximal expiratory flow tion in asthma as determined by maximal expiratory flow
breathing air and a helium-oxygen mixture. $₹$ Clin Invest 1972;51(Suppl 1):3235-43.

14 Gyulay S, Gould D, Sawyer B, Pond D, Mant A, Saunders N. Evaluation of a micro processor based portable home monitoring system to measure breathing during sleep. Sleep 1987;10:130-42.

15 Shepard JW, Garrison MW, Grither DA, Dolan GF Relationship of ventricular ectopy to nocturnal $\mathrm{O}_{2}$ desaturation in patients with obstructive sleep apnoea. Chest 1985;88:335-40.

16 Position Paper of the Thoracic Society of Australia and New Zealand. Treatment of sleep disordered breathing. Thoracic Society News 1990;1:5-8.

17 Ellis E, Bye PT, Bruderer JW, Sullivan CE. Treatment of respiratory failure during sleep in patients with neuromuscular disease. Am Rev Respir Dis 1987;135:148-52.

18 Serisier D, Mastaglia F, Gibson G. Respiratory muscle function and ventillatory control. II Patients with myotonic dystrophy. $Q \mathcal{F}$ Med 1982;202:205-26.

19 Gillam PM. Respiration in myotonic dystrophy. Thorax 1964;19:112-20. 